\title{
Lugares favoritos de adolescentes no Distrito Federal
}

\author{
Isolda de Araújo Günther \\ Gustavo Murici Nepomuceno \\ Mariana Costa Spehar \\ Hartmut Günther \\ Universidade de Brasília
}

\begin{abstract}
Resumo
Explorou-se neste estudo quais os lugares preferidos e não preferidos por 562 jovens (292 F e 270 M), com idades entre 13 e 19 anos (média = 15 anos e 7 meses e desvio padrão = 1 ano e 1 mês), freqüentando a primeira série do ensino médio, sendo 274 de escolas públicas e 288 de escolas particulares. Dentre os participantes 39,5\% moram no Plano Piloto de Brasília e 60,5\% em Cidades Satélites do DF. Em casas, moram 341, em apartamento, 215. Os resultados mostram que o lugar favorito mais freqüentemente mencionado foi a própria casa, indicado por 195 (35,3\%) dos respondentes, seguido por Shopping ( $n=108 ; 19,6 \%)$ e bar/boate/festa ( $\mathrm{n}=103 ; 18,7 \%$ ). O lugar que obteve maior freqüência como não sendo preferido foi bar, boate, festa, indicado por $108(21,3 \%)$ dos participantes, seguido por escola $(n=90 ; 17,7 \%)$ e lugares que provocam sentimentos ruins $(n=32 ; 6,3 \%)$. As diferenças encontradas são discutidas em termos de gênero, tipo de escola (pública ou privada) e tipo de moradia (casa ou apartamento).
\end{abstract}

Palavras-chave: lugares preferidos; identidade de lugar; adolescência; psicologia ambiental

\begin{abstract}
Favorite places of adolescents in the Federal District. This study explored which are the most and least favorite places among 562 adolescents (292 F e $270 \mathrm{M}$ ), with ages between 13 and 19 (mean age $15 \mathrm{yr} 7 \mathrm{mo}$, s.d. 1 yr 1 mo), attending $9^{\text {th }}$ grade, with 274 respondents in public and 288 in private schools. Some $39.5 \%$ live in the central area of Brasília, 60.5\% in the surrounding cities. Some 215 live in apartments, 314 in single occupancy houses. Results indicate that the most frequently mentioned favorite place was the home ( $\mathrm{n}=$ 195, 35.3\%), followed by Shopping Malls $(n=108,19.6 \%)$ and bar/disco/parties $(n=103,18.7 \%)$. The least favorite places were bar/disco/parties $(n=108,21.3 \%)$, school $(n=90,17.7 \%)$ and places that provoke negative feelings $(n=32,6.3 \%)$. Differences are discussed in terms of gender, type of school and type of residence.
\end{abstract}

Key words: favorite places; place identity; adolescence; environmental psychology

A adolescência, além de fenômeno biológico caracterizado por mudanças fisiológicas e hormonais, é fenômeno social modelado por forças culturais e históricas. Este processo dinâmico e recíproco entre forças do desenvolvimento e sócio-culturais, ou seja, mudanças fisiológicas, interesses sexuais emergentes, novos papéis sociais, mudanças de atitude frente aos adultos e dos adultos frente aos jovens força uma redefinição e reavaliação pessoal, um processo denominado de formação da identidade.

Na Psicologia do Desenvolvimento, vários trabalhos hoje clássicos, acrescentaram ao termo identidade outros construtos, como pode ser exemplificado nos estudos sobre difusão e crise de identidade (Erikson, 1968/1972), identidade do eu e status de identidade (Marcia, 1994), ou, mais recentemente, sobre a emergência da identidade do eu (Akers, Jones \& Coyl, 1998).

A Psicologia Ambiental, ao discutir a ligação que as pessoas estabelecem com lugares geográficos, fez uso do conceito de identidade, articulando-o às noções de lugar e apego (attachment), empreendendo estudos sobre identidade de lugar (place-identity) e de apego ao lugar (placeattachment). 
Esta linha de pensamento iniciou-se com Proshansky, Fabian e Kaminoff (1983), ao considerarem que na teorização sobre identidade e conceito de self inexiste uma discussão sobre o papel do ambiente físico. Propuseram, por esta razão, o conceito de identidade de lugar, "teoricamente concebido como aglomerados de cognições com valência positiva e negativa dos ambientes físicos” (p. 74). Identidade de lugar é, portanto, uma estrutura complexa constituída por atitudes, valores, crenças e significados referente à relação psicológica que estabelecemos com os espaços físicos. O lugar foi incluído pelos autores como um subsistema da identidade do eu, cuja particularidade consiste na descrição e socialização da pessoa com o mundo físico. Com esta proposição, Proshansky e colaboradores salientaram que os efeitos dos ambientes físicos são importantes para o desenvolvimento da personalidade por constituírem mais um elemento para definir a pessoa na sociedade. A identidade de lugar desempenha um papel na maneira como o espaço físico é percebido, sentido e significado, por sua vez, o espaço físico ganha significado por meio da identidade de lugar. Transpondo as observações dos autores para a vida cotidiana observamos que muitas das brincadeiras e cantigas infantis trazem conteúdos e rimas sobre lugar. Por outro lado as questões "de onde você veio?”, “você é de onde?” fazem parte do ritual de apresentação das pessoas. Em termos verbais o nome é o primeiro elemento que introduz, mas é o local de procedência que parece completar o imaginário sobre a identidade do outro.

Altman e Low (1992) empregaram o conceito de apego (attachment) ao lugar, enfatizando a relação emocional que estabelecemos com os lugares. Esta noção foi derivada da teoria do apego, a partir do trabalho conjunto de John Bowlby (1969/1984) e Mary Ainsworth (Ainsworth \& Bowlby, 1991). Apego refere-se à ligação afetiva, à busca e à manutenção da proximidade física da criança pequena por sua mãe, bem como à ruptura desta ligação por meio de separação, privação ou morte. A teoria do apego forneceu uma base para teóricos como Kaiser e Fuhrer (1996) reafirmarem que formamos ligações com aqueles ambientes que preenchem nossas necessidades emocionais e que tornam possível o desenvolvimento e manutenção de nossa identidade.

Mais recentemente, Sutton e Kemp (2002) citam Hutchison que considera o ambiente físico como um contexto dinâmico para a vida humana, porque modifica o comportamento, afeta nossos pensamentos, sentimentos, interações sociais, bem-estar físico e self.

Twigger-Ross e Uzzell (1996), a exemplo de Proshansky e colaboradores (1983), concordam que os teóricos que se dedicaram ao estudo do self negligenciaram o ambiente físico. Discordam, entretanto, do argumento de que a identidade de lugar é uma subestrutura da identidade, por ser difícil lidar com "dois aspectos da identidade” (1996, p. 206). Adotar esta dicotomia traria o risco de deslocar o foco da discussão do conceito em si, para a questão de se, ou não, a identidade seria mais social ou lugar. Com base neste argumento, em 1996, após treze anos do trabalho original de Proshansky e colaboradores, Twigger-Ross e Uzzell sugeriram que em vez de ter um componente da identidade relacionado com lugar, "todos os aspectos da identidade terão, em maior ou menor extensão, implicações relacionadas a lugar” (1996, p. 206).

Korpela (2002) deu um passo adiante ao deixar de lado as especulações e separações entre a pessoa, suas cognições, suas emoções e o conceito de lugar. Argumentou que a identidade de lugar é formada pelas experiências e cognições que desenvolvemos nos lugares que desempenham um papel na emoção e na auto-regulação das nossas vidas. Sinalizou para uma integração dos componentes cognitivos e emocionais, isto é, apego ao lugar está implícito em identidade de lugar.

Peña (citado por Deer, 2002) afirmou que lugar é um "repositório primário para as construções humanas de significado e identidade porque os humanos criam significado em parte inscrevendo suas memórias natural e cultural em suas paisagens” (p. 125).

Para a Psicologia Ambiental estudos sobre a identidade de lugar, apego a lugar e temas relacionados como, por exemplo, efeitos restauradores do ambiente, são necessários tanto para o bem-estar psicológico dos indivíduos quanto para a preservação de ambientes e comunidades saudáveis.

No ecletismo dos estudos sobre a criança e o adolescente, um grupo de pesquisadores inspirados nos trabalhos de Vygotsky, Luria e Leontiev conceitualiza o desenvolvimento no seu inter-relacionamento com o contexto externo (Cole,1995; Fuhrer, 1999; Valsiner, 1989). Cultura e desenvolvimento são vistos como uma unidade integral que mutuamente se definem, razão pela qual não podem ser estudados separadamente. Por meio deste efeito recíproco e ativo dos dois lados (Altman \& Rogoff, 1987) indivíduo e cultura se desenvolvem.

O grande desafio, entretanto, é compreender como os jovens negociam e constróem seu desenvolvimento, buscam novas oportunidades, exploram novos papéis, e, no caso deste estudo, estabelecem ligação de apego e desapego com lugares.

Wohlwill (1980), um pioneiro no esforço de convergir a Psicologia do Desenvolvimento e a Psicologia Ambiental, afirmou que teóricos destes dois campos de estudo chegaram à conclusão de que as pessoas agem sobre seus ambientes ao mesmo tempo em que seus ambientes agem sobre as pessoas, modelando seu desenvolvimento. Rivlin, citada por Sutton e Kemp (2002), coloca a questão nestes termos: "Os lugares fornecem abrigo, mas possibilitam uma cola invisível para a experiência humana” (p. 173). É neste sentido que o comentário de Sommer (1990) chama a atenção, quando assim se expressa: "à medida que as crianças vão se desenvolvendo tornam-se conscientes das oportunidades presentes em vários ambientes para satisfazer suas necessidades pessoais” (p. 32). Noack e Silbereisen (1988) apontam na mesma direção quando mostram que os jovens usam seus lugares preferidos como âncora para suas emoções e para a autoregulação de suas vidas.

Quais os resultados das pesquisas que investigam a relação de crianças e jovens com o ambiente? Hart (1979), em 
publicação derivada de sua tese de doutorado, constatou que as preferências das crianças por lugares são determinadas: a) pelo uso do local para brincadeiras; b) pelas pessoas que lá estão; c) pelo que ali podem comprar; d) pela beleza ou sentimento despertado pelo lugar.

Csikszentmihallyi e Larson (1984), interessados em verificar quais os caminhos diários percorridos por setenta e cinco adolescentes, publicaram estudo em que mostravam que suas rotas seguiam três domínios geográficos: a casa, a escola e os lugares públicos - parques, ônibus, supermercados, casa dos amigos. Os autores explicaram que estas rotas devem ser entendidas como três países separados, governados por diferentes leis. Na casa, o adolescente deve buscar um meio de viver com seus pais. Na escola, o adolescente não tem muita chance, senão aceitar as normas da instituição. Nos espaços públicos as regras são variadas e flexíveis. Owens, arquiteta, realizou dois estudos com adolescentes, objetivando conhecer as características dos lugares valorizados pelos jovens para utilizar como base no planejamento de seu trabalho. No primeiro estudo (Owens, 1988), indagou quais os lugares ao ar livre preferidos por 25 jovens californianos entre 14 e 18 anos. A maioria dos respondentes valorizou lugares junto à natureza, seguindo-se lugares em que pudessem estar longe de outras pessoas e, finalmente, lugares em que pudessem ficar com os amigos. No segundo estudo (Owens, 1994), realizou um levantamento de dados com 110 adolescentes australianos e constatou que os lugares mais valorizados pelos adolescentes foram: parques planejados, lugares em casa e áreas comerciais.

Chama a atenção van Andel (1990) para a importância de investigar os lugares de que as crianças gostam, mas também para os lugares de que não gostam, e os que causam medo ou perigo. Realizou um estudo piloto com 36 crianças holandesas entre 6 e 12 anos e um estudo mais amplo com 140 crianças da mesma idade. Os lugares mais mencionados como atrativos foram playgrounds ou áreas verdes. As ruas foram apontadas como os lugares menos atrativos, sendo também consideradas como os mais perigosos.

O presente artigo faz parte de um estudo mais amplo que teve como propósito investigar quais os lugares favoritos de adolescentes. Inspirados na literatura exposta acima, particularmente nas observações de Sommer (1990) ao assentar que "as relações entre lugar e pessoa na modelagem da identidade tornam importante o estudo da preferência por lugares na adolescência” (p. 33) é que este trabalho foi desenvolvido.

\section{Método}

\section{Participantes}

Participaram deste estudo 562 jovens (292 F e 270 M), com idades entre 13 e 19 anos (média de 15 anos e 7 meses, desvio padrão de 1 ano e 1 mês). Os respondentes freqüentavam o primeiro ano do ensino médio em três escolas públicas ( $n=274 ; 48,8 \%$ ) e quatro escolas particulares $(n=288 ; 51,2 \%$ ), uma delas religiosa, do Distrito Federal. No que diz respeito a tipo de moradia, 341 (60,7 \%) moravam em casas, 215 (38,3 \%) em apartamento, cinco não responderam à questão. As análises relacionadas ao número de pessoas na residência, número de quartos na residência e à necessidade de compartilhar o quarto não se revelaram estatisticamente significativas, razão pela qual não serão apresentadas.

\section{Instrumento}

Inspirados no estudo de Sommer (1990), que formulou quatro questões a jovens da Estônia, elaborou-se um instrumento contendo nove questões. De interesse do presente estudo são as perguntas: 1) Qual é o seu lugar preferido? 2) Qual é o lugar em que você não gosta de estar? Solicitaram-se as seguintes informações demográficas para possibilitar a caracterização dos respondentes: sexo, data de nascimento, número de pessoas na residência, local e tipo de residência, se divide, ou não, o quarto com outras pessoas.

\section{Procedimento}

Encaminhou-se uma carta às Coordenadoras das escolas, assinada pelo último autor, solicitando permissão para realizar o estudo. O pedido foi aceito, sem restrições, pelas sete escolas contatadas. Os dados foram coletados pela dupla de pesquisadores júnior - segundo e terceiro autores. O tempo gasto no preenchimento do instrumento variou entre 15 e 20 minutos.

\section{Análise dos dados}

Inicialmente efetuou-se uma análise temática dos enunciados emitidos pelos respondentes. Derivaram-se daí as categorias para a codificação das respostas. Posteriormente, as 113 respostas distintas quanto a lugares favoritos e as 207 quanto a lugares não favoritos foram agrupados em 13 e 25 categorias, respectivamente. Para comparar a freqüência das menções nas diferentes categorias entre grupos por gênero, tipo de escola ou tipo de residência, utilizou-se a análise de qui-quadrado.

\section{Resultados}

\section{Lugares favoritos}

Lugar favorito por gênero. Inicialmente serão apresentadas as preferências por lugares obtidas no estudo, ao mesmo tempo em que será verificado se existe um padrão de similaridades e/ou diferenças quanto ao gênero.

Cento e treze (113) lugares foram mencionados como favoritos pelos respondentes. Estas preferências foram agrupadas em 13 categorias, conforme apresentado na Tabela 1. O lugar mais freqüentemente citado como favorito foi a própria casa, indicado por 195 (35,3 \%) dos adolescentes (95 F e 100 $\mathrm{M}$ ); dentre estes, 31 apontaram o próprio quarto, um, a biblioteca e um, o banheiro. Verifica-se que os 100 (freqüência observada) jovens que indicaram a casa como sendo o lugar preferido, constituem 7,4 respondentes a mais, do que os 92,6 da freqüência esperada pela distribuição que leva em conta a freqüência total dos que indicaram esta opção. Entre as jovens, as 95 que indicaram a casa constituem 7,4 a menos do que a freqüência esperada pela distribuição. Esta diferença 
não é estatisticamente significativa. O segundo lugar declarado como favorito foi o shopping, apontado por 108 (75 F e $33 \mathrm{M})$ dos respondentes. Os 33 jovens que escolheram o shopping como sendo seu lugar preferido constituem 18 a menos do que a freqüência esperada, enquanto que as 75 jovens que assim o fizeram constituem 18 a mais do que o esperado, uma diferença que se mostrou estatisticamente significativa $\left(\stackrel{2}{\circ}_{\text {gl=1 }}=13,83, \mathrm{p}<0,001\right)$. Em seguida, observa-se a preferência para sair à noite para bares, boates e festas, mencionada por 103 dos respondentes. Os 55 jovens que assim o indicaram constituem 6 a mais do que o esperado, enquanto que as 48 jovens representam 6 a menos do que o esperado, uma diferença estatisticamente significativa $\left(\stackrel{\circ}{\circ}_{\mathrm{gl}=1}=8,84\right.$, $\mathrm{p}<0,01)$.
São apontados, em seguida, área verde com 31 (14 F e 17 $\mathrm{M}$ ) indicações, esporte com 26 (14 F e $12 \mathrm{M}$ ), igreja com 22 (12 F e $10 \mathrm{M}$ ), vizinhança com 18 (6 F e $12 \mathrm{M})$ e áreas fora do Distrito Federal com 16 (10 F e $6 \mathrm{M}$ ) indicações. Somente no caso da preferência pela vizinhança, foi ainda verificada uma tendência à significância estatística $\left(\stackrel{.}{\div}_{\mathrm{gl}=1}=2,73, \mathrm{p}<0,10\right)$.

Lugar favorito por moradia. A Tabela 2 apresenta os resultados da relação entre lugares preferidos e o tipo de moradia: casa vs. apartamento. Os resultados apontam duas diferenças estatisticamente significativas: desproporcionalmente mais jovens que residem em casa consideram a casa seu lugar favorito, comparados com os que moram em apartamentos $\left(\div_{\mathrm{gl}=1}^{2}=4,43, \mathrm{p}<0,05\right)$. A outra diferença diz respeito ao shopping, sendo que desproporcionalmente menos jo-

Tabela 1

Freqüências observadas $\left(f_{\text {obs }}\right)$ e esperadas $\left(f_{\text {esp }}\right)$ de lugares favoritos entre adolescentes por gênero.

\begin{tabular}{|c|c|c|c|c|c|c|c|}
\hline \multirow{3}{*}{ Lugar } & \multicolumn{6}{|c|}{ Gênero } & \multirow{3}{*}{$\chi^{2}$} \\
\hline & \multicolumn{2}{|c|}{ Masculino } & \multicolumn{2}{|c|}{ Feminino } & \multicolumn{2}{|c|}{ Total } & \\
\hline & $\mathrm{f}_{\mathrm{obs}}$ & $f_{\text {esp }}$ & $\mathrm{f}_{\text {obs }}$ & $f_{\text {esp }}$ & $\mathrm{N}$ & $\%$ & \\
\hline casa & 100 & 92,6 & 95 & 102,4 & 195 & 35,3 & 1,13 \\
\hline shopping & 33 & 51,3 & 75 & 56,7 & 108 & 19,6 & $13,83 *$ \\
\hline bar/boate/festa & 55 & 48,9 & 48 & 54,1 & 103 & 18,7 & $8,84 * *$ \\
\hline área verde & 17 & 14,7 & 14 & 16,3 & 31 & 5,6 & $<1$ \\
\hline esporte & 12 & 12,3 & 14 & 13,7 & 26 & 4,7 & $<1$ \\
\hline igreja & 10 & 10,4 & 12 & 11,6 & 22 & 4,0 & $<1$ \\
\hline vizinhança & 12 & 8,5 & 6 & 9,5 & 18 & 3,3 & $2,73 * * *$ \\
\hline fora $\mathrm{DF}$ & 6 & 7,6 & 10 & 8,4 & 16 & 2,9 & $<1$ \\
\hline casa de parente & 6 & 4,3 & 3 & 4,7 & 9 & 1,6 & $* * * *$ \\
\hline amigos & 3 & 3,8 & 5 & 4,2 & 8 & 1,4 & \\
\hline escola & 4 & 2,8 & 2 & 3,2 & 6 & 1,1 & \\
\hline cidade DF & 2 & 2,4 & 3 & 2,6 & 5 & 0,9 & \\
\hline lugar cultural & 2 & 2,4 & 3 & 2,6 & 5 & 0,9 & \\
\hline Total $* * * * *$ & $262(4$ & $5 \%)$ & $290(5$ & $, 5 \%)$ & 5 & & \\
\hline
\end{tabular}

Nota

O qui-quadrado para esta tabela como um todo é 21,766, com gl = 12. Entretanto, 10 (38,5\%)

das 26 células (13 lugares x 2 gêneros) têm um valor esperado abaixo de 5 . Diante desta

violação dos requisitos para o cálculo de qui-quadrado, são relatados os componentes do qui-quadrado significativos (Bortz, 1999, p. 169).

* estatisticamente significativo em nível de $\mathrm{p}=0,001, \mathrm{gl}=1$.

** estatisticamente significativo em nível de $\mathrm{p}=0,01, \mathrm{gl}=1$.

$* * *$ estatisticamente significativo em nível de $\mathrm{p}=0,10, \mathrm{gl}=1$.

**** Não foi calculado o valor de $\chi^{2}$ com freqüências esperadas abaixo de 5 .

***** Outros 10 respondentes informaram algum 'outro lugar'. 
Tabela 2

Freqüências observadas $\left(f_{\text {obs }}\right)$ e esperadas $\left(f_{\text {esp }}\right)$ de lugares favoritos entre adolescentes por moradia: casa vs. apartamento.

\begin{tabular}{|c|c|c|c|c|c|c|c|}
\hline \multirow{3}{*}{ Lugar } & \multicolumn{6}{|c|}{ Moradia } & \multirow{3}{*}{$\chi^{2}$} \\
\hline & \multicolumn{2}{|c|}{ Casa } & \multicolumn{2}{|c|}{ Apartamento } & \multicolumn{2}{|c|}{ Total } & \\
\hline & $\mathrm{f}_{\mathrm{obs}}$ & $\mathrm{f}_{\text {esp }}$ & $\mathrm{f}_{\text {obs }}$ & $\mathrm{f}_{\text {esp }}$ & $\mathrm{N}$ & $\%$ & \\
\hline casa & 132 & 117,8 & 60 & 74,2 & 195 & 35,2 & $4,43 *$ \\
\hline shopping & 54 & 65,7 & 53 & 41,3 & 107 & 19,6 & $5,40 *$ \\
\hline Total $* *$ & \multicolumn{2}{|c|}{$335(61,4 \%)$} & \multicolumn{2}{|c|}{$211(38,6 \%)$} & \multicolumn{2}{|l|}{546} & \\
\hline \multicolumn{8}{|c|}{$\begin{array}{l}\text { Nota } \\
\text { Para economizar espaço, são apresentados apenas os dados significativos. Para a lista completa de } \\
\text { lugares preferidos, vide Tabela } 1 \text {. } \\
\text { * estatisticamente significativo em nível de } \mathrm{p}=0,05, \mathrm{gl}=1 \text {. } \\
\text { ** Outros } 16 \text { respondentes ou não informarem a moradia }(\mathrm{n}=6) \text { ou informaram algum 'outro lugar' } \\
(\mathrm{n}=10) \text {. }\end{array}$} \\
\hline
\end{tabular}

vens que moram em casa consideram o shopping como seu lugar favorito, em comparação aos que moram em apartamentos $\left(\stackrel{2}{\mathrm{gl}=1}^{2}=5,40, \mathrm{p}<0,05\right)$.

Lugar favorito e escola. A Tabela 3 apresenta os resultados da relação entre lugares preferidos e a escola freqüentada: particular vs. pública. Os resultados apontam três diferenças estatisticamente significativas: desproporcionalmente mais jovens estudando em escolar particulares consideram o shopping seu lugar favorito, versus relativamente menos alunos de escolas públicas $\left(\div_{\mathrm{gl}=1}^{2}=10,46, \mathrm{p}<0,01\right)$. Por outro lado, desproporcionalmente menos alunos das escolas parti- culares, em comparação aos das escolares públicas, indicam

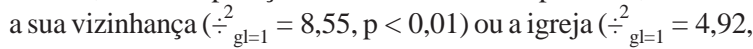
$\mathrm{p}<0,05)$ como sendo um lugar favorito. Verifica-se, ainda, um resultado sugestivo, ainda que não-significativo estatisticamente, no sentido de que proporcionalmente, menos alunos das escolas particulares consideram sua casa como lugar favorito $\left(\stackrel{2}{\circ}_{\mathrm{gl}=1}=3,26, \mathrm{p}<0,10\right)$.

\section{Lugares não preferidos}

Conforme indicado na Tabela 4, os lugares citados como não preferidos foram em número de duzentos e sete (207).

Tabela 3

Freqüências observadas $\left(f_{o b s}\right)$ e esperadas $\left(f_{\text {esp }}\right)$ de lugares favoritos entre adolescentes por escola: particular vs. pública.

\begin{tabular}{|c|c|c|c|c|c|c|c|}
\hline \multirow{3}{*}{ Lugar } & \multicolumn{6}{|c|}{ Escola } & \multirow{3}{*}{$\chi^{2}$} \\
\hline & \multicolumn{2}{|c|}{ particular } & \multicolumn{2}{|c|}{ pública } & \multicolumn{2}{|c|}{ Total } & \\
\hline & $f_{\text {obs }}$ & $f_{\text {esp }}$ & $f_{\text {obs }}$ & $f_{\text {esp }}$ & $\mathrm{N}$ & $\%$ & \\
\hline casa & 87 & 99,6 & 108 & 95,4 & 195 & 35,3 & $3,26 * * *$ \\
\hline shopping & 72 & 55,2 & 36 & 52,8 & 108 & 19,6 & $10,46 *$ \\
\hline igreja & 6 & 11,2 & 16 & 10,8 & 22 & 4,0 & $4,92 * *$ \\
\hline vizinhança & 3 & 9,2 & 15 & 8,8 & 18 & 3,3 & $8,55 *$ \\
\hline Total $* * * *$ & \multicolumn{2}{|c|}{$282(51,1 \%)$} & \multicolumn{2}{|c|}{$270(48,9 \%)$} & \multicolumn{2}{|c|}{552} & \\
\hline
\end{tabular}

Nota

Para economizar espaço, apresentou-se apenas os dados significativos. Para a lista completa de lugares preferidos, vide Tabela 1.

* estatisticamente significativo em nível de $\mathrm{p}=0,01, \mathrm{gl}=1$.

$* *$ estatisticamente significativo em nível de $\mathrm{p}=0,05, \mathrm{gl}=1$.

$* * *$ estatisticamente significativo em nível de $\mathrm{p}=0,10, \mathrm{gl}=1$.

**** Outros 10 respondentes informaram algum 'outro lugar'. 
Tabela 4

Freqüências observadas $\left(f_{\text {obs }}\right)$ e esperadas $\left(f_{\text {esp }}\right)$ de lugares não apreciados entre adolescentes por gênero.

\begin{tabular}{|c|c|c|c|c|c|c|c|}
\hline \multirow{3}{*}{ Lugar } & \multicolumn{6}{|c|}{ Gênero } & \multirow[t]{3}{*}{$\chi^{2}$} \\
\hline & \multicolumn{2}{|c|}{ Masculino } & \multicolumn{2}{|c|}{ Feminino } & \multicolumn{2}{|c|}{ Total } & \\
\hline & $\mathrm{f}_{\mathrm{obs}}$ & $\mathrm{f}_{\text {esp }}$ & $\mathrm{f}_{\mathrm{obs}}$ & $\mathrm{f}_{\text {esp }}$ & $\mathrm{N}$ & $\%$ & \\
\hline bar/boate/festa & 46 & 51,7 & 62 & 56,3 & 108 & 21,3 & 1,21 \\
\hline escola & 61 & 43,1 & 29 & 46,9 & 90 & 17,7 & $14,27 *$ \\
\hline sentimento negativo & 13 & 15,3 & 19 & 16,7 & 32 & 6,3 & $<1$ \\
\hline hospital & 7 & 14,8 & 24 & 16,2 & 31 & 6,1 & $7,87 * *$ \\
\hline casa & 14 & 14,8 & 17 & 16,2 & 31 & 6,1 & $<1$ \\
\hline casa de parente & 15 & 12,0 & 10 & 13,0 & 25 & 4,9 & 1,44 \\
\hline vizinhança & 10 & 9,1 & 9 & 9,9 & 19 & 3,7 & $<1$ \\
\hline cemitério & 5 & 7,7 & 11 & 8,3 & 16 & 3,1 & 1,83 \\
\hline lugar agitado & 4 & 7,2 & 11 & 7,8 & 15 & 3,0 & $2,74 * * * *$ \\
\hline ambiente físico negativo & 5 & 6,7 & 9 & 7,3 & 14 & 2,8 & $<1$ \\
\hline transporte público & 3 & 6,2 & 10 & 6,8 & 13 & 2,6 & $3,16 * * * *$ \\
\hline (alguma) cidade do DF & 8 & 6,2 & 5 & 6,8 & 13 & 2,6 & $<1$ \\
\hline inimigos & 7 & 6,2 & 6 & 6,8 & 13 & 2,6 & $<1$ \\
\hline comércio & 5 & 5,7 & 7 & 6,3 & 12 & 2,4 & $<1$ \\
\hline shopping & 7 & 5,3 & 4 & 5,7 & 11 & 2,2 & $<1$ \\
\hline área verde & 2 & 5,3 & 9 & 5,7 & 11 & 2,2 & $3,97 * * *$ \\
\hline rock & 2 & 4,3 & 7 & 4,7 & 9 & 1,8 & $* * * * *$ \\
\hline fila & 6 & 3,8 & 2 & 4,2 & 8 & 1,6 & \\
\hline esporte & 3 & 3,3 & 4 & 3,7 & 7 & 1,4 & \\
\hline lugar violento & 4 & 3,3 & 3 & 3,7 & 7 & 1,4 & \\
\hline lugar calmo & 4 & 2,9 & 2 & 3,1 & 6 & 1,2 & \\
\hline lugar cultural & 4 & 2,4 & 1 & 2,6 & 5 & 1,0 & \\
\hline igreja & 3 & 2,4 & 2 & 2,6 & 5 & 1,0 & \\
\hline periferia & 3 & 1,9 & 1 & 2,1 & 4 & 0,8 & \\
\hline instituição penal & 2 & 1,4 & 1 & 1,6 & 3 & 0,6 & \\
\hline Total $* * * * * *$ & $243(4$ & $\%)$ & $265(5$ & $\%)$ & 50 & & \\
\hline
\end{tabular}

Nota

O qui-quadrado para esta tabela como um todo é 50,548, com gl = 24. Entretanto, 18 (36\%) das 50 células (25 lugares x 2 escolas) têm um valor esperado abaixo de 5. Diante desta violação dos requisitos para o cálculo de qui-quadrado, são relatados os componentes significativos do qui-quadrado (Bortz, 1999, p. 169).

* estatisticamente significativo em nível de $\mathrm{p}=0,001, \mathrm{gl}=1$.

** estatisticamente significativo em nível de $\mathrm{p}=0,01, \mathrm{gl}=1$.

*** estatisticamente significativo em nível de $\mathrm{p}=0,05, \mathrm{gl}=1$.

$* * * *$ estatisticamente significativo em nível de $\mathrm{p}=0,10, \mathrm{gl}=1$.

$* * * * *$ Não foi calculado o valor de $c^{2}$ com freqüências esperadas abaixo de 5 .

****** Outros 54 respondentes informaram algum 'outro lugar não especifico’. 
Tabela 5

Freqüências observadas $\left(f_{\text {obs }}\right)$ e esperadas $\left(f_{\text {esp }}\right)$ de lugares não apreciados entre adolescentes por moradia: casa vs. apartamento.

\begin{tabular}{|c|c|c|c|c|c|c|c|}
\hline \multirow{3}{*}{ Lugar } & \multicolumn{6}{|c|}{ Moradia } & \multirow{3}{*}{$\chi^{2}$} \\
\hline & \multicolumn{2}{|c|}{ casa } & \multicolumn{2}{|c|}{ apartamento } & \multicolumn{2}{|c|}{ Total } & \\
\hline & $f_{o b s}$ & $f_{\text {esp }}$ & $f_{o b s}$ & $f_{\text {esp }}$ & $\mathrm{N}$ & $\%$ & \\
\hline escola & 46 & 54,5 & 42 & 33,5 & 88 & 17,5 & $3,48 * *$ \\
\hline sentimento negativo & 26 & 19,8 & 6 & 12,2 & 32 & 6,4 & $5,09 *$ \\
\hline casa de parente & 20 & 15,5 & 5 & 9,5 & 25 & 5,0 & $3,44 * *$ \\
\hline Total $* * *$ & \multicolumn{2}{|c|}{$311(62,0 \%)$} & \multicolumn{2}{|c|}{$191(38,0 \%)$} & \multicolumn{2}{|c|}{502} & \\
\hline
\end{tabular}

Nota

Para economizar espaço, apresentou-se apenas os dados significativos. Para a lista completa de lugares nãopreferidos, vide Tabela 4.

* estatisticamente significativo em nível de $\mathrm{p}=0,05, \mathrm{gl}=1$.

** estatisticamente significativo em nível de $\mathrm{p}=0,10, \mathrm{gl}=1$.

*** Outros 60 respondentes ou não informarem moradia $(n=6)$ ou informaram algum 'outro lugar não especifico’ $(n=54)$.

Tais indicações foram agrupadas em vinte e cinco (25) categorias. O lugar indicado como sendo menos preferido (108, 62 F e 46 M) foi sair à noite para ir a bar, boate e festa, não apresentando diferença estatisticamente significativa entre os gêneros. O segundo lugar indicado como menos favorito foi a escola: desproporcionalmente mais rapazes do que moças $\left(\div_{\mathrm{gl}=1}^{2}=14,27, \mathrm{p}<0,001\right)$ fazem esta indicação. Em terceiro lugar segue-se não propriamente um lugar, mas sentimentos negativos relacionados a um lugar, sem haver, entretanto, diferença quanto a gênero. Em seguida, o hospital é mencionado desproporcionalmente em maior número pelas jovens

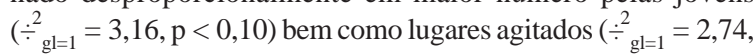
$\mathrm{p}<0,10$ ).

Lugar não preferido por moradia. A Tabela 5 apresenta os resultados da relação entre lugares não preferidos e o tipo de moradia: casa vs. apartamento. Os resultados apontam

Tabela 6

Freqüencias observadas $\left(f_{\text {obs }}\right)$ e esperadas $\left(f_{\text {esp }}\right)$ de lugares não apreciados entre adolescentes por escola: particular vs. pública.

\begin{tabular}{|c|c|c|c|c|c|c|c|}
\hline \multirow{3}{*}{ Lugar } & \multicolumn{6}{|c|}{ Escola } & \multirow{3}{*}{$\chi^{2}$} \\
\hline & \multicolumn{2}{|c|}{ Particular } & \multicolumn{2}{|c|}{ Pública } & \multicolumn{2}{|c|}{ Total } & \\
\hline & $\mathrm{f}_{\mathrm{obs}}$ & $\mathrm{f}_{\mathrm{esp}}$ & $f_{o b s}$ & $f_{\text {esp }}$ & $\mathrm{N}$ & $\%$ & \\
\hline sentimento negativo & 11 & 16,3 & 21 & 15,7 & 32 & 6,3 & $3,51 * *$ \\
\hline casa & 11 & 15,7 & 20 & 15,3 & 31 & 6,1 & $2,85 * *$ \\
\hline vizinhança & 5 & 9,6 & 14 & 9,4 & 19 & 3,7 & $4,46 *$ \\
\hline lugar agitado & 11 & 7,6 & 4 & 7,4 & 15 & 3,0 & $3,08 * *$ \\
\hline shopping & 9 & 5,6 & 2 & 5,4 & 11 & 2,2 & $4,21 *$ \\
\hline Total $* * *$ & 258 & $\%)$ & 250 & $\%)$ & & & \\
\hline
\end{tabular}

Nota

Para economizar espaço, apresentou-se apenas os dados significativos. Para a lista completa de lugares não-preferidos, vide Tabela 4.

* estatisticamente significativo em nível de $\mathrm{p}=0,05, \mathrm{gl}=1$.

** estatisticamente significativo em nível de $\mathrm{p}=0,10, \mathrm{gl}=1$.

*** Outros 54 respondentes informaram algum ‘outro lugar não especifico'. 
uma diferença estatisticamente significativa: desproporcionalmente mais jovens morando em casa consideram lugares que provocam sentimentos negativos como sendo não preferidos $\left(\div_{\mathrm{gl}=1}^{2}=5,09, \mathrm{p}<0,05\right)$. Observa-se, ainda, duas tendências para significâncias estatísticas, com mais moradores de casa indicando não gostar de escola e da casa de parentes.

Lugar não preferido por escola. A Tabela 6 apresenta os resultados da relação entre lugares não preferidos e a escola freqüentada: particular vs. pública. Os resultados apontam duas diferenças estatisticamente significativas no sentido de que desproporcionalmente mais jovens que freqüentam a escola pública indicam gostar menos da sua vizinhança $\left(\div_{\mathrm{gl}=1}^{2}=4,46, \mathrm{p}<0,05\right)$ e dentre os que não gostam de shoppings o maior número freqüenta escolas particulares $\left(\div_{\mathrm{gl}=1}^{2}=4,21, \mathrm{p}<0,05\right)$. Observa-se, ainda, três tendências a diferenças significativas: desproporcionalmente mais alunos de escolas públicas indicam não preferir a própria casa e lugares que provocam sentimentos negativos. Um maior número de alunos de escolas particulares, por sua vez, preferem menos os lugares agitados.

\section{Relação entre lugares preferidos e não preferidos e idade}

Foi observada uma relação significativa entre lugares preferidos e idade (Tabela 7). Entre os jovens de 14 e 15 anos, encontrou-se, desproporcionalmente, maior número indicando os shoppings como lugares preferidos. Entre os com 16 anos ou mais, menos jovens fizeram esta escolha $\left(\div_{\mathrm{gl}=4}^{2}=15,18\right.$, $\mathrm{p}<0,004)$.

\section{Discussão}

Este estudo fundamenta-se no conceito de que a identidade é constituída não apenas pelas relações inter-pessoais e construções sociais. Constitui-se, também, pelas inter-relações que estabelecemos com os lugares significativos para nossa vida, ou seja, o ambiente físico, componente da cultura, parte integrante do desenvolvimento humano (Fuhrer, 1998; Proshansky et al., 1983; Valsiner, 1989).
Estudaram-se lugares, pelo potencial de aglutinar qualidades da pessoa e do ambiente que podem ser vistos como individual ou grupal, interior ou exterior, natural ou construído e por ter a possibilidade de estabelecer um anelo entre o presente, o passado e o futuro (Sebba, 1991). Por sua vez, a identidade de lugar é determinada pelo que a pessoa é, concorre para a auto-definição de quem a pessoa é, uma das tarefas do desenvolvimento na adolescência (Erikson, 1968/ 1972; Havighurst, 1952; Sommer,1990).

Considerando que este componente da cultura, ambiente físico, e o desenvolvimento humano fazem parte de uma unidade que se define mutuamente, investigou-se como adolescentes respondem a duas questões: a) Qual é o seu lugar preferido? b) Qual é o lugar em que você não gosta de estar?

O que primeiro chama a atenção neste estudo é que os lugares preferidos são indicados em menor número que os lugares não preferidos. No estudo de van Andel (1990) a proporção de respostas entre lugares preferidos e não preferidos foi de três lugares preferidos para um lugar não preferido. Comparando estes dois resultados, qual a razão de tamanha diferença? Será decorrente das restrições sociais ligadas à idade que protegem os mais jovens de ambientes não convidativos e, ao mesmo tempo, restringem seu raio de ação? Ou será que, na nossa amostra, os lugares não preferidos estão presentes em maior número na realidade de suas vidas?

Korpela (2002) mostrou que estudos realizados na década de 90 reforçaram a importância das casas, dos ambientes naturais, dos encontros sociais e dos ambientes comerciais como preferidos pelos adolescentes.

No presente estudo a própria casa teve, em números absolutos, a maior indicação. Embora a análise do qui-quadrado tenha indicado que a diferença não é significativa, tal preferência merece um comentário. Por ser a casa o lugar que forma a matriz primária da vida dos indivíduos (Chawla,1991) é de se esperar que seja preferida. Para esta geração, entretanto, a moradia não constituiu o nicho exclusivo do seu desenvolvimento. Muito cedo, a freqüência a creches, escola maternal, jardim de infância, escola de línguas, colônia de férias compe-

Tabela 7

Freqüências observadas $\left(f_{\text {obs }}\right)$ e esperadas $\left(f_{\text {esp }}\right)$ de lugares preferidos entre adolescentes por faixa etária.

\begin{tabular}{|c|c|c|c|c|c|c|c|c|c|c|c|c|c|}
\hline \multirow{3}{*}{ Lugar } & \multicolumn{12}{|c|}{ Faixa etária } & \multirow[t]{3}{*}{$\chi^{2}$} \\
\hline & \multicolumn{2}{|c|}{ até 14 anos } & \multicolumn{2}{|c|}{15 anos } & \multicolumn{2}{|c|}{16 anos } & \multicolumn{2}{|c|}{17 anos } & \multicolumn{2}{|c|}{18 anos } & \multicolumn{2}{|c|}{ Total } & \\
\hline & $f_{o b s}$ & $f_{\text {esp }}$ & $\mathrm{f}_{\mathrm{obs}}$ & $f_{\text {esp }}$ & $\mathrm{f}_{\mathrm{obs}}$ & $f_{\text {esp }}$ & $\mathrm{f}_{\mathrm{obs}}$ & $f_{\text {esp }}$ & $\mathrm{f}_{\mathrm{obs}}$ & $f_{\text {esp }}$ & $\mathrm{N}$ & $\%$ & \\
\hline Shopping & 16 & 9,2 & 66 & 55,3 & 10 & 20,2 & 6 & 10,5 & 5 & 7,8 & 103 & 19,5 & $15,18^{*}$ \\
\hline Total $* *$ & \multicolumn{2}{|c|}{$47(8,9 \%)$} & \multicolumn{2}{|c|}{$284(53,7 \%)$} & \multicolumn{2}{|c|}{$104(19,7 \%)$} & \multicolumn{2}{|c|}{$54(10,2 \%)$} & \multicolumn{2}{|c|}{$40(7,6 \%)$} & \multicolumn{2}{|c|}{529} & \\
\hline
\end{tabular}

Nota

Para economizar espaço, apresentou-se apenas os dados significativos. Para a lista completa de lugares preferidos, vide Tabela 1.

* estatisticamente significativo em nível de $\mathrm{p}<0,004$, gl $=4$.

** Outros 33 respondentes ou não informaram a sua idade $(\mathrm{n}=23)$ ou informaram algum 'outro lugar' $(\mathrm{n}=10)$. 
tiu com este espaço, transformando-o quase em dormitório. Não se pode esquecer, entretanto, de que os jovens precisam de refúgios, de lugares onde é possível restaurar as energias, e experimentar sentimentos de privacidade, segurança e controle. Há melhor lugar para acolher tais sentimentos do que o lar, doce lar?

O shopping foi o lugar mais preferido pelas jovens, pelos que moram em casa e pelos que freqüentam escolas particulares. Em termos de idade, o pico desta preferência se deu entre os quatorze e quinze anos, decaindo gradativamente com o aumento da idade. Estes achados podem ter a seguinte explicação: a preferência por este lugar comercial cresce entre os que parecem ter maior poder aquisitivo e condições econômicas para consumir. Mas será apenas isto? Levando em conta que nove jovens que freqüentam escola particular indicaram o shopping como um lugar menos preferido, a resposta é não.

É, portanto, conveniente interpretar estes achados à luz dos conceitos de behavior-settings (Barker, 1968) ou, mais especificamente, de desenvolvimento como ação em contexto (Noack \& Silbereisen, 1988), ou como lugares para cultivar o desenvolvimento (Fuhrer, 1998). Os dados se tornam mais compreensíveis ao lembrar que dentre as expectativas culturais da adolescência, referidas como tarefas do desenvolvimento (Havighurst, 1952), são sugeridas, entre outras, integração ao grupo de iguais, contato com o sexo oposto e ganho de autonomia.

Fazendo a leitura dos dados a partir deste referencial, a preferência por shopping pode ser compreendida como uma estratégia para o desenvolvimento. Neste espaço comercial com áreas públicas e privadas as regras são variadas e flexíveis. Existe a oportunidade de ocupar o tempo com vários tipos de ação, de lazer, como encontrar os amigos, fazer novas amizades e, assim, auto-regular o próprio desenvolvimento por meio da escolha de um contexto apropriado.

É particularmente interessante a escolha de bar/boate/ festa como lugar preferido por 103 participantes e a escolha do mesmo lugar, como não preferido, por 108 jovens. Esta aparente incongruência se dissolve com este dado de realidade: o espaço vital de alguns adolescentes se caracteriza por atividades peculiares ao seu grupo de idade, a chamada subcultura adolescente. Desde os anos sessenta (Coleman, 1961), sucessivas pesquisas mostraram que alguns jovens aceitam e seguem os valores do seu próprio grupo. Outros, entretanto, adotam os valores dos adultos e exercem atividades bem distintas de sua faixa etária.

A vizinhança foi mais indicada como preferida pelos jovens e como lugar não preferido pelos que freqüentam escolas públicas. O fato dos jovens indicarem preferência pelo ambiente de vizinhança, pode ser uma decorrência dos papéis sociais relacionados ao gênero que os adolescentes são encorajados a adotar. Um dado encontrado por Korpela (2002) refere-se às diferenças entre os gêneros quanto aos tipos de atividades que as crianças escolhem. Os meninos, no estudo citado, tendem a construir seus ambientes de brincadeiras no exterior, ao passo que as meninas tendem a concentrar-se na elaboração e no arranjo do interior dos ambientes. Não encontramos dados similares referentes a adolescentes.
O maior índice de não preferência pela vizinhança pelos adolescentes que freqüentam escolas públicas pode estar relacionado ao crescente sentimento de insegurança e aumento da violência nas áreas residenciais, que envolve até a própria escola.

Verificou-se, também, a não preferência por lugares que despertam sentimentos negativos. Este resultado vai na direção das observações de que fortes emoções estão relacionadas a lugares (Hart, 1979; Sutton \& Kemp, 2002).

O que mais chamou atenção neste estudo foi o número diminuto dos que indicaram preferir a escola (2 F e $4 \mathrm{M}$ ), bem como dos noventa jovens que apontaram a escola como o lugar não favorito. Em estudo anterior (Günther, 1993), o número de jovens que relataram experiência de sucesso na escola foi pequeno. Os dados deste estudo são ainda mais preocupantes e parecem reforçar a opinião de Csikszentmihalyi e Larson (1984) de que a escola, parte prisão, parte templo de aprendizagem tem se tornado "uma máquina que fornece feedback negativo” (p. 198).

Este estudo apenas descortina o vasto mundo das relações entre identidade, lugares preferidos e mecanismos de auto-regulação do desenvolvimento adolescente. Muita coisa resta a ser pesquisada e explicada. Neste ponto compartilhamos do sentimento de Roger Hart (1998) quando assim se expressou: "cientistas sabem provavelmente mais sobre a história natural de outros primatas na selva do que sobre a atividade de crianças [e adolescentes] nas cidades” (p. 2).

\section{Agradecimento}

Os autores agradecem o apoio do CNPq.

\section{Referências}

Ainsworth, M. D. S., \& Bowlby, J. (1991). An ethological approach to personality development. American Psychologist, 46, 331-341.

Akers, J. C., Jones, R. M., \& Coyl, D. D. (1998). Adolescents friendship pairs: similarities in identity status development, behaviors, attitudes, and intentions. Journal of Adolescent Research, 13, 178-201.

Altman, I., \& Low, S. (Orgs.). (1992). Place Attachment. Nova York: Plenum. Altman, I., \& Rogoff, B. (1987). World views in psychology: Trait, interactional, organismic, and transactional perspectives. In D. Stokols \& I. Altman (Orgs.), Handbook of environmental psychology (vol. 1, pp. 7- 40). Nova York: Wiley.

Barker, R. G. (1968). Ecological psychology: Concepts and methods for studying the environments of human behavior. Stanford, Califórnia: Stanford University Press.

Bortz, J. (1999). Statistik für Sozialwissenschaftler [Estatística para cientístas sociais] ( $5^{\mathrm{a}}$ ed.). Berlim: Springer.

Bowlby, J. (1984). Apego (vol. 1, Trilogia Apego e Perda). São Paulo: Martins Fontes (texto original publicado em 1969).

Chawla, L. (1991). Homes for children in a changing society. In E. H. Zube \& G. T. Moore (Orgs.), Advances in Environment, Behavior, and Design (vol. 3, pp. 187-228). Nova York: Plenum.

Cole, M. (1995). Culture and cognitive development: From cross-cultural research to creating systems of cultural mediation. Culture \& Psychology, 1, 25-54. Coleman, J. S. (1961). The adolescent society. Nova York: Free Press.

Csikszentmihalyi, M., \& Larson, R. (1984). Being adolescent: Conflict and growth in the teenage years. Nova York: Basic Books. 
Derr, V. (2002). Children's sense of place in northern New Mexico. Journal of Environmental Psychology, 22, 125-137.

Erikson, E. H. (1972). Identidade juventude e crise. Rio de Janeiro: Zahar. (texto original publicado em 1968).

Fuhrer, U. (1998). Behavior settings as vehicles of children's cultivation. In D. Görlitz, H. J. Harloff, G. Mey \& J. Valsiner (Orgs.), Children, cities, and psychological theories, developing relationships (pp. 411-434). Berlin: de Gruyter.

Günther, I. A. (1993). As necessidades emocionais do adolescente e a escola. Temas em Psicologia, (1), 45-57.

Hart, R. (1979). Children's experience of place. Nova York: Irvington.

Hart, R. (1998). Keynote. In D. Görlitz, H. J. Harloff, G. Mey and J. Valsiner (Orgs.), Children, cities, and psychological theories, developing relationships (pp. 1-4). Berlin: de Gruyter.

Havighurst, R. J.(1952). Developmental task and education. Nova York: David McKay.

Kaiser, F. G., \& Fuhrer, U. (1996). Dwelling: Speaking of an unnoticed universal language. New Ideas in Psychology, 14, 225-236.

Korpela, K. M. (2002). Children’s environment. In R. B. Bechtel \& A. Churchman (Orgs.), Handbook of Environmental Psychology (2⿺ ed., pp. 363-373). Nova York: Wiley.

Marcia, J. E. (1994). The empirical status of ego identity. In H. A. Bosne \& L. G. Tobi (Orgs.), Identity development: An interdisciplinary approach (pp. 67-80). Thousand Oaks, Califórnia: Sage.

Noack, P., \& Silbereisen, R. K. (1988). Adolescent development and choice of leisure settings. Children's Environmental Quarterly, 5(2), 25-32.
Owens, P. E. (1988). Natural landscapes, gathering places, and prospect refuges: characteristics of outdoor places valued by teens. Children's Environments Quarterly, 5(2), 17-24.

Owens, P. E. (1994). Teen places in Sunshine, Australia: Then and now. Children's Environments, 11(4), 292-299.

Proshansky, H., Fabian, A. K., \& Kaminoff, R. (1983). Place-identity: Physical world socialization of the self. Journal of Environmental Psychology, 3, $57-$ 83.

Sebba, R. (1991). The landscapes of childhood. The reflections of childhood's environment in adult memories and in children's attitudes. Environment \& Behavior, 23, 395-422.

Sutton, S. E., \& Kemp, S. P. (2002). Children as partners in neighborhood placemaking: lessons from intergenerational design charrettes. Journal of Environmental Psychology, 22, 171-189.

Sommer, B. (1990). Favorite places of Estonian adolescents. Children's Environmental Quarterly, 7(4), 32-36.

Twigger-Ross, C. L., \& Uzzell, D. (1996). Place and identity processes. Journal of Environmental Psychology, 16, 205-220.

Valsiner, J. (1989). Human development and culture: The social nature of personality and its study. Lexington, Massachusetts: Lexington Books.

van Andel, J. (1990). Places children like, deslike and fear. Children's Environments Quarterly, 7(4), 24-31.

Wohlwill, J. F. (1980). The confluence of environmental and developmental psychology: Signpost to an ecology of development? Human Development, 23, 354-358.

Isolda de Araújo Günther, Ph.D. em Psicologia do Desenvolvimento pela Michigan State University (EUA), é pesquisadora associada sênior no Instituto de Psicologia, UnB (DF) e bolsista do CNPq.

Gustavo Murici Nepomuceno é psicólogo; à época da realização deste trabalho era aluno do Curso de Formação de Psicólogos da UnB e bolsista de Iniciação Científica (CNPq). Mariana Costa Spehar é aluna do Curso de Formação de Psicólogos da UnB e bolsista de Iniciação Científica (CNPq).

Hartmut Günther, Ph.D. em Psicologia Social, University of California at Davis (EUA), é Professor Titular no Instituto de Psicologia, UnB (DF).

Endereço para correspondência: Isolda de Araújo Günther; Caixa Postal 4480; 70919-970; Brasília, DF; Tel.: 61 307.2625, r. 415; E-mail: isolda@unb.br 